Simulations of dynamic crack propagation in brittle materials using nodal cohesive forces and continuum damage mechanics in the distinct element code LDEC

G. I. Block, M. B. Rubin, J. P. Morris, J. G. Berryman

August 23, 2006

International Journal of Fracture 
This document was prepared as an account of work sponsored by an agency of the United States Government. Neither the United States Government nor the University of California nor any of their employees, makes any warranty, express or implied, or assumes any legal liability or responsibility for the accuracy, completeness, or usefulness of any information, apparatus, product, or process disclosed, or represents that its use would not infringe privately owned rights. Reference herein to any specific commercial product, process, or service by trade name, trademark, manufacturer, or otherwise, does not necessarily constitute or imply its endorsement, recommendation, or favoring by the United States Government or the University of California. The views and opinions of authors expressed herein do not necessarily state or reflect those of the United States Government or the University of California, and shall not be used for advertising or product endorsement purposes. 


\section{Simulations of dynamic crack propagation in brittle materials using nodal cohesive forces and continuum damage mechanics in the distinct element code LDEC}

G. Block ${ }^{1,3}$, M.B. Rubin ${ }^{2}$ J. Morris ${ }^{1}$ and J.G. Berryman ${ }^{1,4}$

'Computational Physics Group Atmospheric Earth and Energy Department Lawrence Livermore National Laboratory Livermore, CA 94550

Email: morris50@1lnl.gov

Email: berryman1@1lnl.gov

${ }^{2}$ Faculty of Mechanical Engineering Technion - Israel Institute of Technology 32000 Haifa, Israel

Email: mbrubin@tx.technion.ac.il

${ }^{3}$ Now: Corporate Strategic Research ExxonMobil Research and Engineering 1545 Route 22 East Annandale, NJ 08801

E-mail: gareth.i.block@exxonmobil.com

${ }^{4}$ Now: Geophysics Department, Earth Sciences Division, Lawrence Berkeley National Lab

1 Cyclotron Road MS 90R1116, Berkeley, CA 94720

E-mail: JGBeryman@lbl.gov

Keywords: brittle materials, distinct element, damage, dynamic fracture, cohesive model, Cosserat, effective medium theory 


\begin{abstract}
Experimental data indicates that the limiting crack speed in brittle materials is less than the Rayleigh wave speed. One reason for this is that dynamic instabilities produce surface roughness and microcracks that branch from the main crack. These processes increase dissipation near the crack tip over a range of crack speeds. When the scale of observation (or mesh resolution) becomes much larger than the typical sizes of these features, effective-medium theories are required to predict the coarse-grained fracture dynamics. Two approaches to modeling these phenomena are described and used in numerical simulations. The first approach is based on cohesive elements that utilize a rate-dependent weakening law for the nodal cohesive forces. The second approach uses a continuum damage model which has a weakening effect that lowers the effective Rayleigh wave speed in the material surrounding the crack tip. Simulations in this paper show that while both models are capable of increasing the energy dissipated during fracture when the mesh size is larger than the process zone size, only the continuum damage model is able to limit the crack speed over a range of applied loads. Numerical simulations of straight-running cracks demonstrate good agreement between the theoretical predictions of the combined models and experimental data on dynamic crack propagation in brittle materials. Simulations that model crack branching are also presented.
\end{abstract}




\section{Introduction}

Cohesive elements are based on the pioneering work of Dugdale (1960) and Barenblatt (1962), and have been used for a number of years to model crack growth in brittle materials (e.g., Xu and Needleman, 1994, 1995, 1996; Camacho and Ortiz, 1996, 1997; additional references can be found in Zhou et al., 2005) and in ductile materials (Pandolfi et al., 1999). Fracture is initiated when the traction vector on the common surface between two elements satisfies a specified failure criterion that can depend on both the normal and shear stresses acting on the surface. Typically, when the failure criterion is satisfied, a new cohesive surface element is introduced at this common interface. The cohesive element is used to dissipate energy associated with the formation of new fracture surface by degrading the traction vector applied there.

The computer code LDEC, developed at Lawrence Livermore National Laboratory, is a distinct element code that can model a structure with arbitrary regions of interacting distinct elements and regions of more standard topologically connected finite elements (see Morris et al., 2004, 2005 for more details). The finite element capability is based on the theory of a Cosserat point (Rubin, 1995, 2000) using tetrahedral elements. Recently, the capabilities of LDEC have been enhanced by allowing the finite element region to fracture into distinct elements which can continue to interact at common surfaces, edges, and points.

Standard cohesive models introduce cohesive constitutive equations at common surfaces and finite element discretization techniques transfer these constitutive properties to nodes in the finite element mesh. In contrast, the cohesive model in LDEC is nodal based and is developed for the discretized equations. Specifically, it introduces nodal cohesive forces that dissipate energy due to the fracturing process. This model was 
briefly described in Morris et al. (2005), where preliminary results of fracture were discussed. The objective of this work is to present details of the model and discuss modifications introduced to match experimental data that show limiting crack speeds which are less than the Rayleigh wave speed for a range of applied loads.

In linear elastic fracture mechanics (LEFM), it is known that the Rayleigh wave speed is a limiting value of the crack speed. For example, Liu and Marder (1991) have developed an analytical solution for a crack propagating in an infinite thin strip of finite width which shows that the crack accelerates to the Rayleigh wave speed independent of the value of the strain energy that is stored far ahead of the crack tip. However, experiments by Sharon and Fineberg $(1996,1999)$ using PMMA suggest that a single crack is unstable when it propagates above a critical crack speed. Moreover, they emphasize that the resulting microcracks, which branch off of the main crack, actually move at or near the Rayleigh wave speed. These microcracks lead to increased dissipation that limits the propagation speed of the macrocrack (other dynamical instabilities, which affect the topography of the fracture surface, are responsible for energy dissipation at lower crack speeds). Yoffe's (1951) analytical solution, which shows that the plane of maximum principal stress changes abruptly at higher crack speeds, may suggest one cause of this microcrack branching. In addition, molecular dynamics simulations indicate that the actual crack speed is influenced by softening effects due to nonlinearity in the atomic force potential near failure (Buehler et al., 2003; Abraham, 2005; Buehler and Gao, 2006).

Zhou et al. (2005) analyzed experiments of a propagating edge crack in thin plates of PMMA. The plates were subjected to an extension normal to the crack plane, and the lateral edges of the plate were fixed at a given displacement so that the plate was in 
uniaxial tension before the crack began to move. After performing a large number of experiments, each of which consisted of measuring the crack speed for a given initial (uniaxial) loading, they developed an empirical expression that relates the steady-state crack speed $\mathrm{v}$ to the strain energy per unit area $\mathrm{W}$ in the plate:

$$
\mathrm{W}=\mathrm{G}_{0}\left(\frac{\mathrm{v}_{\infty}}{\mathrm{v}_{\infty}-\mathrm{v}}\right),
$$

where $\mathrm{v}_{\infty}=675 \mathrm{~m} / \mathrm{s}$ and $\mathrm{G}_{0}=1000 \mathrm{~N} / \mathrm{m}$ are constants given in Zhou et al. (2005). When (1.1) is rewritten in terms of the applied stress [noting that $\mathrm{W}=\mathrm{H} \sigma^{2} /(2 \mathrm{E})$, where $\mathrm{E}$ is Young's modulus and $\mathrm{H}$ is the plate height], their fit shows that as $\sigma$ approaches the static tensile strength $\sigma_{\mathrm{c}}$, the crack speed $\mathrm{v}$ increases to $\mathrm{v}_{\infty}$, which is approximately $74.5 \%$ of the Rayleigh wave speed $\mathrm{v}_{\mathrm{R}}=609 \mathrm{~m} / \mathrm{s}$.

In LEFM, the fracture energy released by a steady-state crack should exactly balance the loss of strain energy far ahead of the crack tip. Zhou et al. (2005) therefore interpreted (1.1) to mean that the effective surface energy increases as the velocity increases. To model this increased dissipation, they proposed a rate-dependent modification of the usual linear weakening law used in cohesive elements. While the functional form is phenomenological, it can be used to predict more accurately the limiting values of the crack speed observed at the lower stress levels in their experiments. At stress levels close to the fracture strength of PMMA, their rate-dependent model again predicts that the crack speed approaches the Rayleigh wave speed if the simulation is restricted to allow for only a single crack surface. However, when their simulations allow for macroscopic crack branching (using a mesh size that is comparable to the process zone size), the crack velocity based on the tip of the most advanced branch compares well with their experimental date for crack speeds in thin plates. 
Sharon and Fineberg (1999) report maximum micro-branch lengths of about $1.5 \mathrm{~mm}$ in PMMA, which is smaller than the macro-branches observed in the simulations of Zhou et al. (2005); the simulated fracture patterns are also quite different from those observed in the experiments. Because of these issues, it is questionable whether the speed of the most advanced macro-branch is representative of the limiting speed of the macrocrack. In this work, Sharon and Fineberg's (1999) conclusions are interpreted to mean that above a critical crack speed the microcracks (propagating at the Rayleigh wave speed) cause the macrocrack to propagate into a pre-cracked, damaged region. Consequently, the macrocrack should propagate at the reduced Rayleigh wave speed associated with the effective moduli of the pre-damaged material ahead of its tip. This effect is modeled here by introducing continuum damage of the element constitutive equations. As will be discussed in later sections, while it is possible to use cohesive elements as a mechanism for upscaling when the mesh size is larger than the process zone, a combination of both continuum damage and rate-dependent cohesive element models may be required to simulate the macroscopic behavior of dynamic fracture. 


\section{General equations for a nodal cohesive force}

The distinct element code LDEC has been designed to handle regions of distinct tetrahedral elements that are free to interact through contact of surfaces, edges and points, as well as regions of tetrahedral elements, which maintain common nodes as in standard finite element methods. The elements are treated as Cosserat points (Rubin, 1995, 2000), which can experience homogeneous deformations and whose kinematics and constitutive equations are fully nonlinear. In particular, the constitutive equations can be used to simulate shock loading since they model coupled nonlinear thermomechanical response with viscoplasticity. However, only nonlinear elastic response with brittle failure is considered in this work.

Cohesive element models typically focus attention on failure of the contact stress at the common surface between two neighboring elements. In contrast, the model of fracture proposed here (and implemented in LDEC) focuses attention on the failure of the cohesive bond between nodes instead of between surfaces-it is therefore called a nodal cohesive force model. When the average stress at a node reaches a specified failure criterion, the node is broken into two daughter nodes (see Fig. 1) and the force acting between the daughter nodes is degraded to dissipate an amount of energy related to the new fracture surface area being created. Details of this procedure are described below.

The Cosserat equations, which describe the motion of the $\mathrm{I}^{\text {th }}$ element, can be written in the nodal forms

$$
\frac{1}{4}{ }_{\mathrm{I}}{ }_{\mathrm{I}} \dot{\overline{\mathbf{w}}}_{\mathrm{i}}={ }_{\mathrm{I}} \mathrm{m}_{\mathrm{I}} \overline{\mathbf{b}}^{\mathrm{i}}-{ }_{\mathrm{I}} \overline{\mathbf{t}}^{\mathrm{i}}+{ }_{\mathrm{I}} \overline{\mathbf{m}}^{\mathrm{i}}
$$

where $\mathrm{i}=0,1,2,3$. Here, ${ }_{\mathrm{I}} \mathrm{m}$ is the mass of the element, ${ }_{\mathrm{I}} \overline{\mathbf{w}}_{\mathrm{i}}$ is the velocity of the $\mathrm{i}^{\text {th }}$ node, ${ }_{\mathrm{I}}^{\mathrm{m}} \overline{\mathrm{b}}^{\mathrm{i}}$ model the effects of body forces, ${ }_{\mathrm{I}} \overline{\mathbf{t}}^{\mathrm{i}}$ are intrinsic forces which require constitutive 
equations, and ${ }_{\mathrm{I}} \overline{\mathbf{m}}^{\mathrm{i}}$ are external forces applied to the element at its nodes. Note that (2.1) uses a lumped mass approximation.

Next, consider the case when the $\mathrm{K}^{\text {th }}$ node breaks into two daughter nodes which are located by the position vectors $\mathbf{x}_{\mathrm{A}}^{*}$ and $\mathbf{x}_{\mathrm{B}}^{*}$. Let $\mathbf{f}_{\mathrm{A} / \mathrm{B}}$ be the nodal cohesive force applied by the node $\mathbf{x}_{\mathrm{B}}^{*}$ on the node $\mathbf{x}_{\mathrm{A}}^{*}$ and let $\mathbf{f}_{\mathrm{B} / \mathrm{A}}$ be the nodal cohesive force applied by the node $\mathbf{x}_{\mathrm{A}}^{*}$ on the node $\mathbf{x}_{\mathrm{B}}^{*}$. The equations of motion of the daughter nodes can be written in the forms

$$
\sum_{\mathrm{A} ; \mathrm{I} ; \mathrm{i}}\left[{ }_{\mathrm{I}} \overline{\mathbf{m}}^{\mathrm{i}}\right]=\mathbf{f}_{\mathrm{A} / \mathrm{B}}, \quad \sum_{\mathrm{B} ; \mathrm{I} ; \mathrm{i}}\left[{ }_{\mathrm{I}} \overline{\mathbf{m}}^{\mathrm{i}}\right]=\mathbf{f}_{\mathrm{B} / \mathrm{A}}=-\mathbf{f}_{\mathrm{A} / \mathrm{B}},
$$

where the special summation symbol indicates summation over all elements I and all nodes $\mathrm{i}$ which have the common node A or B. It is convenient to introduce the effective masses $\mathrm{m}_{\mathrm{A}}$ and $\mathrm{m}_{\mathrm{B}}$ and the forces $\mathbf{F}_{\mathrm{A}}$ and $\mathbf{F}_{\mathrm{B}}$ by the expressions

$$
\begin{gathered}
\mathrm{m}_{\mathrm{A}}=\sum_{\mathrm{A} ; \mathrm{I}}\left[\frac{1}{4} \mathrm{I} \mathrm{m}\right], \quad \mathrm{m}_{\mathrm{B}}=\sum_{\mathrm{B} ; \mathrm{I}}\left[\frac{1}{4} \mathrm{I} \mathrm{m}\right], \\
\mathbf{F}_{\mathrm{A}}=\sum_{\mathrm{A} ; \mathrm{I} ; \mathrm{i}}\left[{ }_{\mathrm{I}} \mathrm{m}_{\mathrm{I}} \overline{\mathbf{b}}^{\mathrm{i}}-{ }_{\mathrm{I}} \overline{\mathbf{t}}^{\mathrm{i}}\right], \quad \mathbf{F}_{\mathrm{B}}=\sum_{\mathrm{B} ; \mathrm{I} ; \mathrm{i}}\left[{ }_{\mathrm{I}} \mathrm{m}_{\mathrm{I}} \overline{\mathbf{b}}^{\mathrm{i}}-{ }_{\mathrm{I}} \overline{\mathbf{t}}^{\mathrm{i}}\right],
\end{gathered}
$$

where the summation for the mass quantities is over all elements I that have a common node with A or B. Thus, the equations of motion (2.2) can be written in the forms

$$
\mathrm{m}_{\mathrm{A}} \ddot{\mathbf{x}}_{\mathrm{A}}^{*}=\mathbf{F}_{\mathrm{A}}+\mathbf{f}_{\mathrm{A} / \mathrm{B}}, \quad \mathrm{m}_{\mathrm{B}} \ddot{\mathbf{x}}_{\mathrm{B}}^{*}=\mathbf{F}_{\mathrm{B}}+\mathbf{f}_{\mathrm{B} / \mathrm{A}} \text {. }
$$

At the instant that the node $\mathrm{K}$ breaks, the nodes $\mathrm{A}$ and $\mathrm{B}$ coincide, the accelerations $\ddot{\mathbf{x}}_{\mathrm{A}}^{*}$ and $\ddot{\mathbf{x}}_{\mathrm{B}}^{*}$ are equal and the cohesive force $\mathbf{f}_{\mathrm{A} / \mathrm{B}}$ has the initial value $\mathbf{f}_{0}$ given by the solution of the equation 


$$
\frac{\mathbf{F}_{\mathrm{A}}+\mathbf{f}_{0}}{\mathrm{~m}_{\mathrm{A}}}=\frac{\mathbf{F}_{\mathrm{B}}-\mathbf{f}_{0}}{\mathrm{~m}_{\mathrm{B}}},
$$

which yields

$$
\mathbf{f}_{\mathrm{A} / \mathrm{B}}=\mathbf{f}_{0}=\frac{\mathrm{m}_{\mathrm{A}} \mathbf{F}_{\mathrm{B}}-\mathrm{m}_{\mathrm{B}} \mathbf{F}_{\mathrm{A}}}{\mathrm{m}_{\mathrm{A}}+\mathrm{m}_{\mathrm{B}}}
$$

at fracture initiation.

The fracturing process causes the daughter nodes $\mathbf{x}_{\mathrm{A}}^{*}$ and $\mathbf{x}_{\mathrm{B}}^{*}$ to separate, with the distance $\delta$ between the nodes and the unit direction vector $\mathbf{e}_{\mathrm{B} / \mathrm{A}}$ being defined by

$$
\delta^{2}=\left(\mathbf{x}_{\mathrm{B}}^{*}-\mathbf{x}_{\mathrm{A}}^{*}\right) \cdot\left(\mathbf{x}_{\mathrm{B}}^{*}-\mathbf{x}_{\mathrm{A}}^{*}\right), \quad \mathbf{e}_{\mathrm{B} / \mathrm{A}}=\frac{1}{\delta}\left(\mathbf{x}_{\mathrm{B}}^{*}-\mathbf{x}_{\mathrm{A}}^{*}\right), \quad \mathbf{e}_{\mathrm{B} / \mathrm{A}} \cdot \mathbf{e}_{\mathrm{B} / \mathrm{A}}=1
$$

Then, the nodal cohesive force is given by $\mathbf{f}_{\mathrm{A} / \mathrm{B}}=-\mathbf{f}_{\mathrm{B} / \mathrm{A}}=\mathrm{f} \mathbf{e}_{\mathrm{B} / \mathrm{A}}$, where

$$
\mathrm{f}=\mathrm{f}_{0}(1-\omega) \frac{\delta}{\delta_{\max }}
$$

is the weakening law, $\delta_{\max }$ is the historical maximum of $\delta$ over the lifetime of the element, and $\mathrm{f}_{0}=\left|\mathbf{f}_{0}\right|$. The singularity when $\delta_{\max }$ vanishes is removed by setting the ratio $\delta / \delta_{\max }$ equal to unity for $\delta_{\max }=0$. Also, in order to maintain continuity of the nodal cohesive force at the instant of cracking the value of $\mathbf{e}_{\mathrm{B} / \mathrm{A}}$ is specified by

$$
\mathbf{e}_{\mathrm{B} / \mathrm{A}}=\frac{\mathbf{f}_{0}}{\mathrm{f}_{0}},
$$

at fracture initiation.

A critical feature of (2.8) is the degradation parameter $\omega$, which characterizes the amount of damage that occurs on the microscale in a region about each daughter node. Once the value of $\omega$ equals unity, the nodal cohesive force vanishes and the cohesive element is removed from the calculation (except in the case of granddaughter nodes, 
which is discussed in Appendix A). While the degradation parameter satisfies $0 \bullet \omega \bullet 1$ in general, its evolution is determined by an equation that depends on the model used (specific examples are discussed in Section 4).

Since failure criteria are typically formulated in terms of stress conditions, it is convenient to define ${ }_{\mathrm{K}} \mathbf{T}_{\text {avg }}$ as the volume averaged Cauchy stress in the elements that connect to node $\mathrm{K}$ :

$$
{ }_{\mathrm{K}} \mathbf{T}_{\mathrm{avg}}=\frac{\sum_{\mathrm{K}}{ }_{{ }^{\mathrm{d}}{ }_{\mathrm{I}}{ }^{1 / 2} \mathbf{T}}}{\sum_{\mathrm{K}}{ }_{\mathrm{d}^{1 / 2}{ }_{\mathrm{I}}^{\mathrm{V}}}},
$$

where the summation is taken over all elements connected to node $\mathrm{K},{ }_{\mathrm{I}}{ }^{1 / 2}{ }_{\mathrm{I}} \mathbf{T}$ is a tensor in the Cosserat theory that is directly related to the volume averaged Cauchy stress in the $\mathrm{I}^{\text {th }}$ element and $\mathrm{I}^{\mathrm{d}}{ }^{1 / 2} \mathrm{I}^{\mathrm{V}}$ is the current volume of the $\mathrm{I}^{\text {th }}$ element. The constitutive equation for the Cosserat element causes ${ }_{\mathrm{K}} \mathbf{T}_{\text {avg }}$ to separate additively into a part ${ }_{\mathrm{K}} \hat{\mathbf{T}}_{\text {avg }}$ due to elastic response and a part ${ }_{\mathrm{K}}{ }^{\mathrm{V}}$ avg due to linear viscous damping:

$$
{ }_{\mathrm{K}} \mathbf{T}_{\text {avg }}={ }_{\mathrm{K}} \hat{\mathbf{T}}_{\text {avg }}+{ }_{\mathrm{K}} \stackrel{\mathrm{v}}{\text { avg }} .
$$

The work of Camacho and Ortiz $(1996,1997)$ uses a failure criterion that includes the effects of both normal and shear stresses. Here, the simplest maximum tensile stress criterion is considered. Specifically, using the elastic part ${ }_{\mathrm{K}} \hat{\mathbf{T}}_{\text {avg }}$ of the average stress it is possible to determine the principal values $\sigma_{\mathrm{i}}$ of this elastic part of the stress and the associated principal directions $\mathbf{p}_{\mathrm{i}}$ such that 


$$
\sigma_{1} \cdot \sigma_{2} \cdot \sigma_{3}
$$

For example, when $\sigma_{1}$ first equals the failure stress $\sigma_{\mathrm{c}}$ the node $\mathrm{K}$ is split into two daughter exterior nodes. The neighboring elements that were connected to the node $\mathrm{K}$ then adopt one of the daughter nodes as their new node K. This node is determined by passing a plane normal to the principal direction $\mathbf{p}_{1}$ through the node $\mathrm{K}$. Each element adopts the daughter that lies on the same side of the plane as its centroid. This process is sketched in Fig. 1 for a nodal fracture in two dimensions. If a daughter node breaks into granddaughter nodes before the nodal cohesive force applied to it vanishes, the cohesive force must be split into two new nodal cohesive forces, as described in Appendix A.

It is also mentioned that this cohesive nodal force formulation uses collinear forces between connected nodes so that there is no influence on the global balance of angular momentum. In contrast, cohesive surface elements that allow for shear stresses acting on surfaces with finite separation (as in Camacho and Ortiz, 1996, 1997; Pandolfi et al., 1999) should influence the global angular momentum of the body being modeled. 


\section{Energy dissipated by the nodal cohesive force}

In order to analyze the rate of material dissipation caused by the cohesive force, it is convenient to introduce the rate of work $W_{c}$ done on the nodal cohesive element and the strain energy $V_{c}$ in the cohesive element by the expressions

$$
\mathrm{W}_{\mathrm{c}}=\left(-\mathbf{f}_{\mathrm{A} / \mathrm{B}}\right) \cdot \dot{\mathrm{x}}_{\mathrm{A}}^{*}+\left(-\mathbf{f}_{\mathrm{B} / \mathrm{A}}\right) \cdot \dot{\mathbf{x}}_{\mathrm{B}}^{*}, \quad \mathrm{~V}_{\mathrm{c}}=\frac{1}{2} \mathrm{f} \delta=\frac{1}{2} \mathrm{f}_{0}(1-\omega) \frac{\delta^{2}}{\delta_{\max }} .
$$

Since the cohesive element has no kinetic energy, the rate of dissipation $D_{c}$ in the cohesive element can be expressed in the form

$$
\mathrm{D}_{\mathrm{c}}=\mathrm{W}_{\mathrm{c}}-\dot{\mathrm{V}}_{\mathrm{c}} \cdot 0
$$

which is required to be nonnegative for cracking to be dissipative. Moreover, with the help of (2.7) and (2.8), it follows that

$$
\mathrm{D}_{\mathrm{c}}=\mathrm{f} \dot{\delta}-\dot{\mathrm{V}}_{\mathrm{c}}=\frac{1}{2} \mathrm{f}_{0} \frac{\delta}{\delta_{\max }}\left[(1-\omega) \frac{\delta}{\delta_{\max }} \dot{\delta}_{\max }+\delta \dot{\omega}\right],
$$

where use has been made of fact that $\mathrm{f}_{0}$ is constant. Since $\left\{\delta, \delta_{\max }, \dot{\delta}_{\max },(1-\omega)\right\}$ are nonnegative, it follows that the restriction

$$
\dot{\omega} \cdot 0
$$

is a sufficient condition for the dissipation (3.3) to be nonnegative.

For the simplest rate-independent model, the maximum crack opening displacement $\delta_{\mathrm{c}}$ is constant and the degradation parameter is given by:

$$
\omega=\operatorname{Min}\left[\frac{\delta_{\max }}{\delta_{c}}, 1\right]
$$

which is the integral of the evolution equation 


$$
\dot{\omega}=\frac{\dot{\delta}_{\max }}{\delta_{c}}, \text { with } \omega(0)=0
$$

and which satisfies the inequality (3.4). In this case, the functional form of the magnitude of the cohesive force $\mathrm{f}$ in (2.8), as shown in Fig. 2, is similar to that used by Camacho and Ortiz $(1996,1997)$.

Let $A_{c}$ be the reference area associated with the crack surface that is created by the failure (including both faces of new surface area exposed by creation of the crack) and let $\gamma$ be the energy per unit reference surface area associated with creating new crack surface. Equating the total energy dissipated by the cohesive force to the crack surface energy for the rate-independent case yields

$$
\frac{1}{2} \mathrm{f}_{0} \delta_{\mathrm{c}}=\mathrm{A}_{\mathrm{c}} \gamma
$$

Thus, the critical opening displacement $\delta_{c}$ of the cohesive node is given by

$$
\delta_{\mathrm{c}}=\frac{2 \mathrm{~A}_{\mathrm{c}} \gamma}{\mathrm{f}_{0}}
$$

Although cracking initiates when the average nodal stress equals the specified value $\sigma_{c}$, it is important to emphasize that the magnitude $\mathrm{f}_{0}(2.6)$ of the nodal cohesive force, the area $\mathrm{A}_{\mathrm{c}}$ of new fracture surface, and the magnitude $\delta_{\mathrm{c}}(3.8)$ that are associated with the crack opening displacement are determined at the onset of cracking. They therefore depend on the current geometry of the elements and the orientation of the plane that is perpendicular to the principal direction of stress. Because these quantities are determined by equations that relate the energy dissipated in cracking to the new surface area being formed, $\left\{\sigma_{c}, \gamma\right\}$ are material constants and $\left\{\mathrm{f}_{0}, \delta_{\mathrm{c}}, \mathrm{A}_{\mathrm{c}}\right\}$ are auxiliary variables in the model. 


\section{A rate-dependent model}

The simulations in Zhou et al. (2005) were based on a cohesive element that depends on both the shear and normal stresses applied to the cohesive surface; the form of the effective cohesive force was similar to that of the nodal force in (2.8) with the specification (3.5) for the degradation parameter $\omega$. They showed that for a rateindependent cohesive element (with constant $\delta_{c}$ ), the crack velocity approached the Rayleigh wave speed asymptotically as the stress $\sigma$ was increased. In an attempt to model the experimental results described by (1.1), Zhou et al. (2005) introduced a ratedependent cohesive law that connects the crack opening displacement parameter $\delta_{\mathrm{c}}$ to the rate of crack opening $\dot{\delta}_{\text {eff }}$ :

$$
\delta_{\mathrm{c}}=\delta_{\mathrm{c} 0}\left[1+\left\{\frac{\dot{\delta}_{\mathrm{eff}}}{\dot{\delta}_{0}}\right\}^{\mathrm{n}}\right]
$$

where $\left\{\delta_{\mathrm{c} 0}, \dot{\delta}_{0}, \mathrm{n}\right\}$ are material constants [the term $\delta_{\mathrm{c} 0}$ is defined by (3.8)]. Complete fracture occurs in their model (and the cohesive element is deleted) when the criterion $\delta_{\text {eff }}=\delta_{\mathrm{c}}$ is satisfied.

In order to limit the computational effort involved in simulating the full-scale problem, Zhou et al. (2005) performed simulations of a scaled-down version of the plates that were subjected experimentally to uniaxial stress $\sigma$ normal to the crack plane. As depicted in Fig. 3, the plates' lateral boundaries were fixed, while boundaries on all other surfaces were free. This same geometry is used in the simulations discussed in the following sections. 
The main effect of the constitutive equation (4.1) proposed by Zhou et al. (2005) is to cause the rate of dissipation of the cohesive element to increase with increasing crack opening rate. This is a phenomenological model for the physical processes that lead to surface roughness and branching, which increase the effective surface area of the crack as the speed increases (and thus increase the rate of dissipation for a given velocity of the macroscale crack tip). The results of Zhou et al. (2005) for the rate-independent (RI) and rate-dependent (RD) cases are depicted in Figure 4. As is discussed in the following sections, while rate-dependent formulations are able to show reasonable agreement with the experimental data for the lower stress levels, they seem to be less adequate for the higher stress levels associated with the limiting crack speed.

Within the context of the present nodal cohesive force model, a functional form similar to (4.1) could be specified by

$$
\delta_{\mathrm{c}}=\delta_{\mathrm{c} 0}\left[1+\left(\alpha \dot{\delta}_{\mathrm{max}} / \delta_{\mathrm{c} 0}\right)^{\mathrm{n}}\right]
$$

where $\left\{\delta_{\mathrm{c} 0}, \alpha, \mathrm{n}\right\}$ are constants and (3.5) is used for $\omega$. One modification in (4.2) is the replacement of $\dot{\delta}_{\text {eff }}$ in (4.1) by $\dot{\delta}_{\text {max }}$ to ensure that the rate dependence occurs only during inelastic loading. The second modification is the normalization of $\dot{\delta}_{\max }$ by the values $\delta_{\mathrm{c} 0}$, which accounts for the fact that the value of $\delta_{\mathrm{c} 0}$ is a different constant for each nodal cohesive force. The value of $\alpha$ that is consistent with both the model (4.2) and the values used in the simulations in Zhou et al. (2005) becomes

$$
\alpha=\frac{\delta_{\mathrm{c} 0}}{\dot{\delta}_{0}}=\frac{8 \times 10^{-6} \mathrm{~m}}{5 \mathrm{~m} / \mathrm{s}}=1.6 \times 10^{-6} \mathrm{~s}
$$


However, it should be mentioned that the functional forms in (4.1) and (4.2) do not necessarily ensure that the rate of dissipation satisfies the inequality (3.2).

To overcome some of the difficulties inherent in the Zhou et al (2005) formulation, a phenomenological model has been developed that modifies the rate-of-change of the degradation parameter $\omega$ in (2.8). One example is the form:

$$
\begin{gathered}
\dot{\omega}=\langle 1-\alpha \dot{\varepsilon}\rangle^{\mathrm{n}} \dot{\varepsilon} \\
\varepsilon=\frac{\delta_{\max }}{\delta_{\mathrm{c}}}, \dot{\varepsilon}=\frac{\dot{\delta}_{\max }}{\delta_{\mathrm{c}}}, \mathrm{n} \cdot 0, \quad \alpha \cdot 0, \quad \omega(0)=0,
\end{gathered}
$$

where $\{\alpha, \mathrm{n}\}$ are constants, $\delta_{\mathrm{c}}$ is a constant given by (3.8), and the Macaulay brackets are defined by

$$
\langle x\rangle=\frac{1}{2}(x+|x|)
$$

The dissipation inequality is satisfied automatically because the degradation parameter $\omega$ increases monotonically during the fracture process.

For low values of rate $(\alpha \dot{\varepsilon}<<1)$ and monotonic loading $\left(\dot{\delta}_{\max }>0\right)$, the total energy dissipated by the nodal cohesive force is approximately equal to the surface energy released during fracture of the mesh [as in (3.7)]. However, when $(\alpha \dot{\varepsilon})$ approaches unity, $\dot{\omega}$ vanishes and the nodal cohesive force remains constant during loading. The total energy dissipated is then approximately $\mathrm{f}_{0} \delta_{\max }$ (with energy continually being dissipated by the cohesive element until $\omega=1)$. As the crack accelerates, the rate of opening $\dot{\delta}_{\max }$ 
tends to increase monotonically until $\dot{\omega}=0$, after which $\dot{\delta}_{\text {max }}$ oscillates about (and decays towards) a steady-state value that dictates the asymptotic crack speed. 


\section{Damage evolution in the process zone}

The rate-dependent model described in Section 4 introduces added dissipation once the failure criterion has been satisfied and daughter nodes are created. However, the material ahead of the crack tip has not yet failed and therefore remains undamaged. It will be shown in the next section that this model of degradation of nodal cohesive forces is able to affect the limiting value of crack speed observed in experiments at lower stress levels, but not at higher stress levels. In this section, a phenomenological model of damage is proposed that increases dissipation at the higher stress levels (presumably associated with microcracking in the process zone surrounding the crack tip). For simplicity, an isotropic damage model is considered that degrades the strain energy $\Sigma^{*}$ of the continuum to the value $\Sigma$ such that

$$
\Sigma=(1-\Omega) \Sigma^{*}
$$

where $\Omega$ is a damage variable that is determined by integrating an evolution equation. Assuming that $\Sigma^{*}$ remains non-negative, this functional form will satisfy the second law of thermodynamics provided that $\dot{\Omega}$ is non-negative. Since the damage model in (5.1) is included as part of the constitutive relation in each tetrahedra in LDEC, it is completely separate from the cohesive element algorithms described previously.

The value of $\Omega$ is determined by integrating an evolution equation, one example of which is:

$$
\dot{\Omega}=\beta_{1}\left\langle\Omega_{\mathrm{d}}-\Omega\right\rangle, \quad \Omega(0)=0, \beta_{1} \bullet 0,
$$

where $\Omega_{\mathrm{d}}$ is a function of stress specified by

$$
\Omega_{\mathrm{d}}=\left[\frac{\beta_{2} \omega_{\mathrm{d}}}{1+\beta_{2} \omega_{\mathrm{d}}}\right] \Omega_{\mathrm{d} 0}, \quad \omega_{\mathrm{d}}=\left\langle\frac{\sigma_{1}-\beta_{3} \sigma_{\mathrm{c}}}{\left(1-\beta_{3}\right) \sigma_{\mathrm{c}}}\right\rangle,
$$




$$
0 \bullet \Omega_{\mathrm{d} 0}<1, \quad \beta_{2} \bullet 0, \quad 0<\beta_{3}<1
$$

In these equations, $\left\{\beta_{1}, \beta_{2}, \beta_{3}, \Omega_{\mathrm{d} 0}\right\}$ are constitutive constants. The rate of evolution of $\Omega$ is controlled by the constant $\beta_{1}$, while its maximum value $\Omega_{\mathrm{d}}$ is a stress-dependent quantity that depends on the constants $\left\{\beta_{2}, \Omega_{\mathrm{d} 0}\right\}$ and on the function $\omega_{\mathrm{d}}$. Also, the stress-dependent value of $\omega_{\mathrm{d}}$ ensures that damage does not increase when the maximum principal stress $\sigma_{1}$ (due to the elastic part of the average stress tensor in the element) is less than or equal to the value $\beta_{3} \sigma_{c}$. The functional forms in (5.2) and (5.3) were chosen because they capture a number of the characteristic features of evolving damage; they are also robust enough to allow for reasonable fits to experimental data. 


\section{LDEC Simulations}

A number of simulations have been performed using a PMMA plate (shown in Fig. 3) whose dimensions were similar to those used in the Zhou et al. (2005) simulations; the plate was thinner and smaller than the samples used in their experiments, but with approximately the same aspect ratio. Appendix B describes the stress-strain relation used for elements in LDEC, as well as a derivation of the parameters needed to set up a uniaxial stress field in the simulations.

The plate had a reference length $\mathrm{L}$, height $\mathrm{H}$, and thickness $\mathrm{T}$ :

$$
\mathrm{L}=32 \mathrm{~mm}, \mathrm{H}=16 \mathrm{~mm}, \mathrm{~T}=0.5 \mathrm{~mm} \text {. }
$$

The material properties of PMMA used in the simulations were:

$$
\begin{gathered}
\rho_{0}=1.180 \mathrm{Mg} / \mathrm{m}^{3}, \quad E=3.09 \mathrm{GPa}, \quad v=0.35, \\
\sigma_{\mathrm{c}}=75 \mathrm{MPa}, \quad \mathrm{v}_{\mathrm{R}}=0.906 \mathrm{~km} / \mathrm{s}, \quad \gamma=150 \mathrm{~N} / \mathrm{m},
\end{gathered}
$$

where $\rho_{0}$ is the mass density, $E$ is Young's modulus, $v$ is Poisson's ratio, $\sigma_{c}$ is the static value of uniaxial stress tensile strength and $v_{R}$ is the Rayleigh wave speed for PMMA, all associated with the small deformation theory. The plate had a pre-existing edge crack in the shape of a triangular wedge with height $\mathrm{a}=4 \mathrm{~mm}$ and base $\mathrm{b}=0.2 \mathrm{~mm}$.

The element mesh consisted of homogeneously deformable tetrahedral elements, and two mesh sizes were used to explore potential mesh dependence of the results. The typical lengths of the elements for the coarsest mesh (denoted by CM, with 17,084 tetrahedra and 5,326 nodes) and the more refined mesh (denoted by RM, with 44,872 tetrahedra and 11,605 nodes) are:

$$
500 \mu \mathrm{m} \text { for } \mathrm{CM}, \quad 250 \mu \mathrm{m} \text { for RM. }
$$


The coarse and refined meshes had one and two elements through the plate thickness, respectively. While the process zone size is approximately $150 \mu \mathrm{m}$ in PMMA, use of these mesh sizes is consistent with our view that both the cohesive element and continuum damage models are capable of modeling the macroscale behavior of fracture, as long as the model coefficients are understood to be based on an underlying effectivemedium theory.

All of the simulations used the formulation (2.8) for degradation of the nodal cohesive forces. The degradation parameter $\omega$ and the damage variable $\Omega$ evolved according to the functional forms described by (4.4) and (5.1), respectively. The parameters used in these functional forms were determined by fitting the LDEC simulation results to the Zhou et al. (2005) empirical relation (1.1) through a combination of trial and error and rough estimation. For rate-independent (RI) and rate-dependent (RD) degradation of the nodal cohesive forces, $\mathrm{n}=0.2$ and $\alpha$ was specified by

$$
\alpha=0(\mathrm{RI}), \quad \alpha=9.35 \times 10^{-8} \mathrm{~s}(\mathrm{RD})
$$

For those simulations that include continuum damage, the values of $\beta_{\mathrm{i}}$ were specified by

$$
\beta_{1}=5 \times 10^{6} \mathrm{~s}^{-1}, \quad \beta_{2}=10, \quad \beta_{3}=0.95
$$

Also, for those simulations that omit the damage mechanism, $\beta_{1}$ is set to zero. One of the benefits of the formalism in (5.1) is that the limiting value $\Omega_{\mathrm{d} 0}$ can be determined from the value of the limiting crack speed at the highest stress levels. Specifically,

$$
\Omega_{\mathrm{d} 0}=1-\left(\frac{\mathrm{v}_{\infty}}{\mathrm{v}_{\mathrm{R}}}\right)^{2} \approx 0.445,
$$

since the elastic wave speeds are proportional to the square root of the moduli. 
Results from LDEC simulations of both straight and branching cracks are shown in Figs. 5-10. Simulations of crack branching allowed all nodes in the mesh to break if the criterion (2.13) was met, so that the fracture was free to find its optimum path through the mesh. Simulations of straight cracks were set up so that only nodes along the mid-plane of the plate were allowed to break. For these calculations, instead of using the maximum principal value of stress for the failure criterion, the value of $\sigma_{1}$ was specified by the value of stress acting normal to the fracture plane with unit normal $\mathbf{n}$, such that [see (B.4) for details]

$$
\sigma_{1}=\mathbf{n} \cdot\left({ }_{\mathrm{K}} \hat{\mathbf{T}}_{\mathrm{avg}} \mathbf{n}\right)
$$

For each run, the nodes of the tetrahedral element were initially deformed by the deformation field (B.6) to produce a uniform initial stress field $\sigma$. At $t=0$, the prenotched edge crack was allowed to run to its steady-state value v; steady state was always reached well before the crack reached the far side of the plate. Crack speeds were computed using the slope of a plot of the crack-tip position versus time, which attained its asymptotic value within 10 microseconds for all runs.

Note that the surfaces of the crack are stress free so that wave propagation starts immediately near these surfaces. As the stress relief waves propagate outwards, the stress intensity at the crack tip increases and failure occurs, which causes the crack to move. In this regard, it should be mentioned that the simulations in Zhou et al. (2005) initialized the dynamic crack propagation problem using deformations in the mesh that were obtained by solving for the static solution of a crack of length a that was not initially allowed to propagate. It is expected that the near steady-state propagation of the crack is relatively unaffected by these differences in the initialization procedures. 
A comparison of the empirical fit (1.1) to LDEC simulations for the rate-independent (RI) and rate-dependent (RD) cases is shown in Fig. 5 for both coarse (CM) and refined (RM) meshes. These simulations examine the effect of degradation of the nodal cohesive force only (2.8) and omit continuum damage (5.1). Although rate dependence of the degradation of the nodal cohesive force improves the predictions for the lower stress levels, the crack speed continues to approach the Raleigh wave speed at the higher stress levels. This result is consistent with an extrapolation of the results of Zhou et al. (2005), which are shown in Fig. 4. One explanation is that the cohesive models alone cannot dissipate enough energy (when branching is prevented) to balance the loss of the strain energy stored near the crack tip. Indeed, if (1.1) is interpreted as in Zhou et al. (2005), the effective surface energy should increase without bound as the stress increases-for this to occur, the cohesive element would need to stay active (and continue to dissipate energy) for longer periods than the functional form (4.4) allows.

A comparison of the empirical fit (1.1) to LDEC simulations for the rate-independent (RI) and rate-dependent (RD) cases without damage is shown in Fig. 6a and with damage is shown in Fig. 6b for both coarse (CM) and refined (RM) meshes. In contrast with the rate-dependent cohesive element formulations in (4.2) and (4.4), damage models are capable of lowering the limiting crack speeds even at the highest stress levels. Furthermore, it is noted that combining the damage model with rate-dependent cohesive elements was required to predict the strain energy-velocity curve over the entire experimental range.

Two issues arise with the damage model for the smallest applied loads. First, damage may cause the crack to arrest when using the coarsest mesh: as depicted in Fig. 6a and Fig. 6b, two simulations resulted in zero crack speed when the load decreased below a 
critical value. Second, there is intrinsic mesh-size dependence in the results because mesh refinement causes the energy dissipation potential of the cohesive nodal forces to dominate that of continuum damage. To understand this process, consider a typical cubical region with edge length $\mathrm{L}$, which is meshed by $\mathrm{n}^{3}$ equal cubical regions, each of which is meshed by five tetrahedral elements. Assuming uniaxial stress of value $\sigma$ and using the constitutive equations (5.1) - (5.3) it can be shown that the total energy that can be dissipated by continuum damage is approximated by

$$
\mathrm{U}_{\mathrm{d}}=\Omega_{\mathrm{d} 0}\left(\frac{\sigma^{2}}{2 \mathrm{E}}\right) \mathrm{L}^{3}
$$

On the other hand, for rate-independent response (3.7) the total energy that can be dissipated by the cohesive nodal forces is given by

$$
\mathrm{U}_{\mathrm{c}}=\gamma\left[2 \mathrm{n}^{3} \alpha_{\mathrm{t}}\left(\frac{\mathrm{L}^{2}}{\mathrm{n}^{2}}\right)\right]=\gamma\left(2 \mathrm{n} \alpha_{\mathrm{t}}\right) \mathrm{L}^{2}, \quad \alpha_{\mathrm{t}}=12.93
$$

where $\alpha_{t} L^{2}$ represents the sum of the surface areas of the five tetrahedral elements that mesh the cubical region when $n=1$. Consequently,

$$
\frac{\mathrm{U}_{\mathrm{c}}}{\mathrm{U}_{\mathrm{d}}}=\mathrm{n}\left[\frac{4 \mathrm{E} \gamma \alpha_{\mathrm{t}}}{\Omega_{\mathrm{d} 0} \sigma^{2} \mathrm{~L}}\right],
$$

which suggests that for refined meshes (large values of $\mathrm{n}$ ) branching will tend to dominate continuum damage as the main dissipative mechanism of fracture, whereas for coarse meshes continuum damage makes a significant contribution to dissipation during the fracturing process.

In accordance with LEFM, it is expected that for high applied loads the crack speed should approach a vertical asymptote-the "damaged" Rayleigh wave speedcharacterized by $\Omega_{\mathrm{d} 0}$. This scenario is exhibited by the RI-RM case in Fig. 6a. However, 
as the RD-RM case in Fig. $6 \mathrm{~b}$ suggests, a very good fit over the entire load range can be obtained by combining both the damage model and rate-dependent cohesive elements, whose surface-energy contribution increases when the mesh size approaches that of the process zone size.

Figure 7 shows the maximum principal stress $\sigma_{1}$ and Fig. 8 shows the damage $\Omega$ for propagation of a single fracture using the rate-independent degradation of the nodal cohesive force (4.4) and the continuum damage model, which are indistinguishable from the rate-dependent cases. Snapshots for different loads and at different times are presented for the refined mesh (RM). One feature that arises (and made especially clear by constraining the crack along the mid-plane) is that the maximum stress points in directions that are off-axis from the crack for rapidly moving fractures [in line with the result due to Yoffe (1951)]. Figure 8 also indicates that the Yoffe-type instability affects predictions of the proposed stress-based continuum damage model.

Macroscopic branching can be simulated when fracture is allowed to occur at any node in the mesh. These simulations also made use of the rate-independent degradation of the nodal cohesive force and the continuum damage model; the rate-dependent cases are expected to be similar. Figure 9 shows the maximum principal stress $\sigma_{1}$ and Fig. 10 shows the damage $\Omega$ predicted during macroscopic branching of the main fracture. Snapshots for different loads and at different times are presented for the refined mesh (RM). While the patterns appear to be qualitatively accurate, further research is necessary to determine whether LDEC is capable of predicting the observables that might serve as reliable metrics of large-scale branching (e.g., acoustic emission spectra, etc.). 


\section{Discussion}

This paper has focused on the development of constitutive relations for use in the code LDEC that capture the macroscale behavior of crack propagation in brittle materials. Because the branching process increases dissipation near the crack tip over a range of crack speeds, additional mechanisms are required to ensure that macroscale cracks will propagate at the correct speed in numerical simulations. When the scale of observation (or mesh resolution) becomes much larger than the typical sizes of microbranching observed experimentally, effective-medium theories are required to predict the coarse-grained fracture dynamics. Two approaches to modeling these phenomena have been described and used in numerical simulations. The first approach is based on cohesive elements that utilize a rate-dependent weakening law for the nodal cohesive forces. The second approach uses a continuum damage model which lowers the effective Rayleigh wave speed in the material surrounding the crack tip.

Both of the approaches described here- a class of rate-dependent weakening laws for cohesive elements and a continuum damage model—have demonstrated the ability to reproduce certain features of the experimental data for straight cracks. The combined model using both of these approaches compares well with the empirical fit (1.1) proposed by Zhou et al.'s (2005) over the entire experimental range of loading.

A number of issues distinguish this work from previous research in the area of fracture modeling. Simulations performed at scales smaller than the process zone size typically assume that the surface energy released during fracture is a well-defined material property, and therefore constant. If branching occurs, the total energy dissipated increases automatically as additional surface area is created. Cohesive elements then describe processes at the scale of the mesh, while continuum damage of an element might 
be used to describe features that exist at smaller scales. When the scale of observation (or mesh size) is larger than the process zone size, small-scale phenomena are necessarily lumped into constitutive relations defined over a volume or an interface (or between interfacial nodes, as in the present implementation of cohesive elements). Effectivemedium relations-including those used here-are valid only at a given scale of observation, and simulations based on them should produce mesh size-dependent features that are consistent with this scale.

While comparing the empirical fit (1.1) to the speeds of straight cracks (as in the previous section) is a useful goal for developing an intuition about the role of various functional forms and model parameters, future work needs to focus on the issue of adapting the models for a given scale of observation. In particular, if the Yoffe instability is the main cause of branching in the simulations, the effective-medium coefficients that are used must somehow inhibit this process by affecting the local stress field as an emergent feature of the model. Simulations that allow branching should produce straight cracks for large mesh sizes (over a range of length scales for which the experimentalist cannot discern micro-branching), but branching cracks at smaller sizes. (Note that "convergence" as the mesh size decreases to zero has a completely different meaning here.) Robust simulations of such scale-dependent (or equivalently, mesh-dependent) behaviors and across-scale coupling are critical to enhancing deeper understanding of fracture and other nonlinear systems. 


\section{Appendix A. Creation of granddaughter nodes}

As described in Section 2, if a daughter node breaks into granddaughter nodes before the nodal cohesive force applied to it vanishes, then this cohesive force must be split into two new nodal cohesive forces. Specifically, let $\left\{\mathrm{m}_{1}, \mathrm{~m}_{2}\right\}$ be the masses of the new granddaughter nodes and let $\left\{\mathrm{f}_{0}, \delta, \delta_{\max }, \omega\right\}$ be the values associated with the nodal cohesive force at the instant of creation of the granddaughter nodes. Also, let $\left\{\mathrm{A}_{\mathrm{c} 1}, \mathrm{~A}_{\mathrm{c} 2}\right\}$ be the portions of the area $A_{c}$ (such that $A_{c}=A_{c 1}+A_{c 2}$ ) that were associated with the initiation of the fracture but are now associated with the granddaughter nodes.

At the instant of creation of the granddaughter nodes, the nodal cohesive forces associated with the granddaughter nodes have the same direction as the parent nodal cohesive force. Moreover, the values $\left\{\delta, \delta_{\max }, \omega\right\}$ of the granddaughters are the same as those of the parents, but the values $\left\{\mathrm{f}_{01}, \mathrm{f}_{02}\right\}$ of the magnitudes of the new nodal cohesive forces and the values $\left\{\delta_{\mathrm{c} 1}, \delta_{\mathrm{c} 2}\right\}$ of their critical opening displacement are defined by the expressions

$$
\mathrm{f}_{01}=\left[\frac{\mathrm{m}_{1}}{\mathrm{~m}_{1}+\mathrm{m}_{2}}\right] \mathrm{f}_{0}, \quad \delta_{\mathrm{c} 1}=\frac{2 \gamma \mathrm{A}_{\mathrm{c} 1}}{\mathrm{f}_{01}}, \mathrm{f}_{02}=\left[\frac{\mathrm{m}_{2}}{\mathrm{~m}_{1}+\mathrm{m}_{2}}\right] \mathrm{f}_{0}, \quad \delta_{\mathrm{c} 2}=\frac{2 \gamma \mathrm{A}_{\mathrm{c} 2}}{\mathrm{f}_{02}} .
$$

These expressions were developed so that at the instant the granddaughter nodes are created, the total force applied by the new nodal cohesive forces is the same as that of the parent:

$$
\mathrm{f}_{01}+\mathrm{f}_{02}=\mathrm{f}_{0} .
$$

Thus, the contributions to the acceleration of the granddaughter nodes of the new nodal cohesive forces are the same: 


$$
\frac{f_{01}}{m_{1}}=\frac{f_{02}}{m_{2}},
$$

and the energy dissipated by the new nodal cohesive forces is the same as what would have been dissipated by the parent nodal cohesive force

$$
\frac{1}{2} \mathrm{f}_{01} \delta_{\mathrm{c} 1}+\frac{1}{2} \mathrm{f}_{02} \delta_{\mathrm{c} 2}=\frac{1}{2} \mathrm{f}_{0} \delta_{\mathrm{c}}=\mathrm{A}_{\mathrm{c}} \gamma
$$




\section{Appendix B. Deformation gradient for uniaxial stress}

Each element in LDEC is characterized by a nonlinear isotropic elastic strain energy function, which characterizes the constitutive equations of the elements. Recall that a material point $\mathbf{X}$ in the fixed stress-free reference configuration is deformed to the point $\mathbf{x}$ in the present configuration, and that the three dimensional deformation measures $\{\mathbf{F}, \mathrm{J}$, $\left.\mathbf{B}, \alpha_{1}\right\}$ are defined by

$$
\mathbf{F}=\cdot \mathbf{x} / \cdot \mathbf{X}, \quad \mathrm{J}=\operatorname{det}(\mathbf{F}), \quad \mathbf{B}=\mathbf{F F}^{\mathrm{T}}, \quad \alpha_{1}=\mathrm{J}^{-2 / 3} \mathbf{B} \cdot \mathbf{I},
$$

where $\mathbf{I}$ is the unit tensor, $\mathbf{A} \cdot \mathbf{B}=\operatorname{tr}\left(\mathbf{A B}^{\mathrm{T}}\right)$ denotes the scalar product between two second order tensors $\{\mathbf{A}, \mathbf{B}\}$ and $\alpha_{1}$ is a pure measure of distortional deformation, which is based on the work of Flory (1961). Next, the strain energy function is proposed in the form

$$
\rho_{0} \Sigma=(1-\Omega)\left[\mathrm{K}\{(\mathrm{J}-1)-\ln (\mathrm{J})\}+\frac{1}{2} \mu\left(\alpha_{1}-3\right)\right],
$$

where $\rho_{0}$ is the reference mass density, $K$ is the bulk modulus and $\mu$ is the shear modulus associated with the small deformation response of the undamaged $(\Omega=0)$ material

$$
\mathrm{K}=\frac{\mathrm{E}}{3(1-2 v)}, \quad \mu=\frac{\mathrm{E}}{2(1+v)} .
$$

It then follows that the Cauchy stress $\mathbf{T}^{*}$ associated with (7.3) is given by

$$
\mathbf{T}^{*}=(1-\Omega)\left[-\mathrm{K}\left(\frac{1}{\mathbf{J}}-1\right) \mathbf{I}+\mu \mathrm{J}^{-5 / 3}\left\{\mathbf{B}-\frac{1}{3}(\mathbf{B} \cdot \mathbf{I}) \mathbf{I}\right\}\right] .
$$

This functional form determines the elastic part of the average stress in each element. The linear viscous part of the average stress in each element is discussed in Morris et al. (2004) and is characterized by normalized damping coefficients $\zeta_{1}$ for dilatational deformation rate and $\zeta_{2}$ for distortional deformation rate which are specified by 


$$
\zeta_{1}=\zeta_{2}=0.1
$$

For the initial conditions of the simulations, the plate is taken to be undamaged $(\omega=\Omega=0)$ and at rest with the deformation field being specified by

$$
\mathrm{x}_{1}=\lambda \mathrm{X}_{1}, \quad \mathrm{x}_{2}=\mathrm{b} \mathrm{X}_{2}, \quad \mathrm{x}_{3}=\mathrm{b} \mathrm{X}_{3}
$$

Here, and throughout the text, the components of all tensors are referred to the fixed rectangular Cartesian base vectors $\mathbf{e}_{\mathrm{i}}$, with $\left\{\mathrm{x}_{\mathrm{i}}, \mathrm{X}_{\mathrm{A}}, \mathrm{T}_{\mathrm{ij}}\right\}$ being the components of $\{\mathbf{x}, \mathbf{X}$, $\mathbf{T}\}$, respectively. Also, for uniaxial stress $\sigma$ in the $\mathbf{e}_{1}$ direction of the undamaged material the stretches $\{\lambda, b\}$ are determined by solving the equations

$$
\begin{gathered}
\sigma=-\mathrm{K}\left[\frac{1}{\mathrm{~J}}-1\right]+\frac{2 \mu}{3} \mathrm{~J}^{-5 / 3}\left(\lambda^{2}-\mathrm{b}^{2}\right), \quad J=a b^{2}, \\
K\left[\frac{1}{\mathrm{~J}}-1\right]+\frac{\mu}{3} \mathrm{~J}^{-5 / 3}\left(\lambda^{2}-\mathrm{b}^{2}\right)=0 .
\end{gathered}
$$

Combining these equations the values of $\{\mathrm{J}, \mathrm{b}\}$ can be determined by

$$
J=\frac{1}{1-\frac{\sigma}{3 \mathrm{~K}}}, \quad \mathrm{~b}=\sqrt{\frac{\mathrm{J}}{\lambda}},
$$

where for $0 \bullet \sigma<3 \mathrm{~K}, \lambda$ is the positive real solution of the cubic equation

$$
\lambda^{3}-\left[\frac{\sigma}{\mu \mathrm{J}^{-5 / 3}}\right] \lambda-\mathrm{J}=0 .
$$




\section{Acknowledgements}

Part of this work was supported by UC, LLNL G\&A account of the Energy and Environment Directorate and the U.S. Department of Energy under Contract No. W7405-ENG-48. The authors would like to thank E. Herbolzheimer, T.Thomas Halsey, T_arabay Antoun, and L.ew Glenn for their support and thoughtful discussions. 


\section{References}

Abraham, F.F. (2005). Unstable crack motion is predictable. Journal of the Mechanics and Physics of Solids 53, 1071-1078.

Barenblatt, G.I. (1962). The mathematical theory of equilibrium of cracks in brittle fracture. Advances in Applied Mechanics 8, 55-129.

Buehler, M., Abraham, F.F. and Gao, H. (2003). Hyperelasticity governs dynamic fracture at a critical length scale. Nature 426, 141-146.

Buehler, M. and Gao, H. (2006). Dynamical fracture instabilities due to local hyperelasticity at crack tips. Nature $\mathbf{4 3 9}, 307-310$.

Camacho, G.T. and Ortiz, M. (1996). Computational modeling of impact damage in brittle targets. International Journal of Solids and Structures 33, 2899-2938.

Camacho, G.T. and Ortiz, M. (1997). Adaptive Lagrangian modeling of ballistic penetration of metallic targets. Computer Methods in Applied Mechanics and Engineering 142, 269-301.

Dugdale, D.S. (1960). Yielding of steel sheets containing slits. Journal of the Mechanics and Physics of Solids 8, 100-104.

Fineberg, J., Gross, S.P., Marder, M. and Swinney, H.L. (1991). Instability in dynamic fracture. Physical Review Letters 67, 457-460.

Fineberg, J., Gross, S.P., Marder, M. and Swinney, H.L. (1992). Instability in the propagation of fast cracks. Physical Review B 45, 5146-5154.

Flory, P. (1961). Thermodynamic relations for high elastic materials. Transactions of the Faraday Society 57, 829-838.

Liu, X. and Marder, M. (1991). The energy of a steady-state crack in a strip. Journal of the Mechanics and Physics of Solids 39, 947-961. 
Morris, J.P., Rubin, M.B., Blair, S.C., Glenn, L.A. and Heuze, F.E. (2004). Simulations of underground structures subjected to dynamic loading using the distinct element method. Engineering Computations 21, 384-408.

Morris, J.P., Rubin, M.B., Block, G.I. and Bonner, M.P. (2005). Simulations of Fracture and Fragmentation of Geologic Materials using Combined FEM/DEM Analysis. To appear in Journal of Impact Engineering.

Pandolfi, A., Krysl, P, and Ortiz, M. (1999). Finite element simulations of ring expansion and fragmentation: The capturing of length and time scales through cohesive models of fracture. International Journal of Fracture 95, 279-297.

Rubin, M.B. (1995). Numerical solution of two- and three-dimensional thermomechanical problems using the theory of a Cosserat point, Journal of Math. and Physics (ZAMP) 46, Special Issue, S308-S334. In Theoretical, Experimental, And Numerical Contributions To The Mechanics Of Fluids And Solids, Edited by J. Casey and M. J. Crochet, Brikhauser Verlag, Basel (1995).

Rubin, M.B. (2000). Cosserat Theories: Shells, Rods and Points, Solid Mechanics and its Applications, Vol. 79, Kluwer, The Netherlands.

Sharon, E. and Fineberg, J. (1996). Microbranching instability and the dynamic fracture of brittle materials. Physical Review B 54, 7128-7139.

Sharon, E. and Fineberg, J. (1999). Confirming the continuum theory of dynamic brittle fracture for fast cracks. Nature 397, 333-335.

Xu, X-P. and Needleman, A. (1994). Numerical simulations of fast crack growth in brittle solids. Journal of the Mechanics and Physics of Solids 42, 1397-1434. 
Xu, X-P. and Needleman, A. (1995). Numerical simulations of dynamic interfacial crack growth allowing for crack growth away from the bond line. International Journal of Fracture 74, 253-275.

Xu, X-P. and Needleman, A. (1996). Numerical simulations of dynamic crack growth along an interface. International Journal of Fracture 74, 289-324.

Yoffe, E.H. (1951). The moving Griffith crack. Philosophical Magazine 42, 739-750.

Zhou, F., Molinari, J.F. and Shioya, T. (2005). Rate-dependent cohesive model for simulating dynamic crack propagation in brittle materials. Engineering Fracture Mechanics 72, 1383-1410. 


\section{List of figures}

Fig. 1 Sketch of nodal fracture in two dimensions.

Fig. 2 Sketch of the response of the cohesive force given in (2.8) with the specification (3.5).

Fig. 3 Sketch of the simulated specimen.

Fig. 4 Empirical fit to experimental data for PMMA together with data from Zhou et al. (2005) for rate-independent (RI) and rate-dependent (RD) models.

Fig. 5 Empirical fit to experimental data for PMMA together with simulations using rate-independent $(\mathrm{RI})$ and rate-dependent $(\mathrm{RD})$ degradation of the nodal cohesive force with no continuum damage evolution. Also shown are the results for a coarse mesh $(\mathrm{CM})$ and a more refined mesh $(\mathrm{RM})$.

Fig. 6 Empirical fit to experimental data for PMMA together with simulations using rate-independent $(\mathrm{RI})$ and rate-dependent $(\mathrm{RD})$ degradation of the nodal cohesive force with damage evolution. Also shown are the results for a coarse mesh $(\mathrm{CM})$ and a more refined mesh $(\mathrm{RM})$.

Fig. 7 Maximum principal stress $\sigma_{1} / \sigma_{\mathrm{c}}$ for a single fracture using the refined mesh (RM): Snapshots at 1, 15, and 30 microseconds after initial loading to $60 \%$ and $90 \%$ of tensile strength (left and right panels, respectively).

Fig. 8 Damage $\Omega$ for a single fracture using the refined mesh (RM): Snapshots at 1 , 15, and 30 microseconds after initial loading to $60 \%$ and $90 \%$ of tensile strength (left and right panels, respectively).

Fig. 9 Maximum principal stress $\sigma_{1} / \sigma_{\mathrm{c}}$ for branching using the refined mesh (RM):

Snapshots at 1, 15, and 30 microseconds after initial loading to $60 \%$ and $90 \%$ of tensile strength (left and right panels, respectively). 
Fig. 10 Damage $\Omega$ for branching using the refined mesh (RM): Snapshots at 1,15 , and 30 microseconds after initial loading to $60 \%$ and $90 \%$ of tensile strength (left and right panels, respectively). 


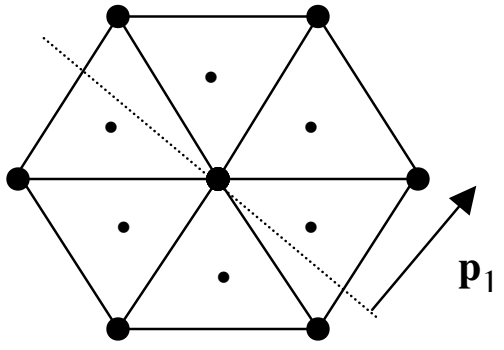

before fracture

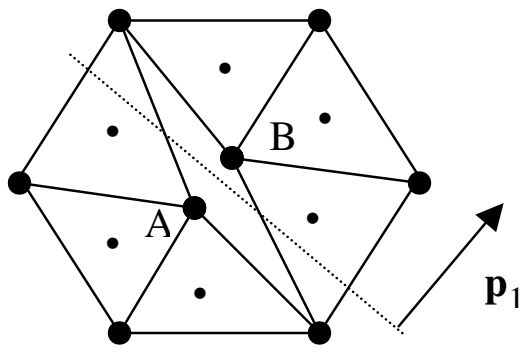

after fracture

Fig. 1 Sketch of nodal fracture in two dimensions. 


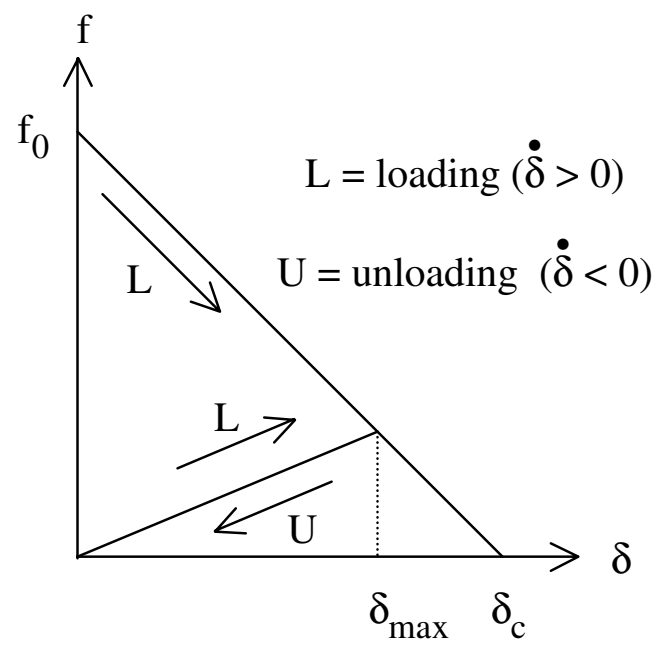

Fig. 2 Sketch of the response of the nodal cohesive force given in (2.8) with the specification (3.5). 


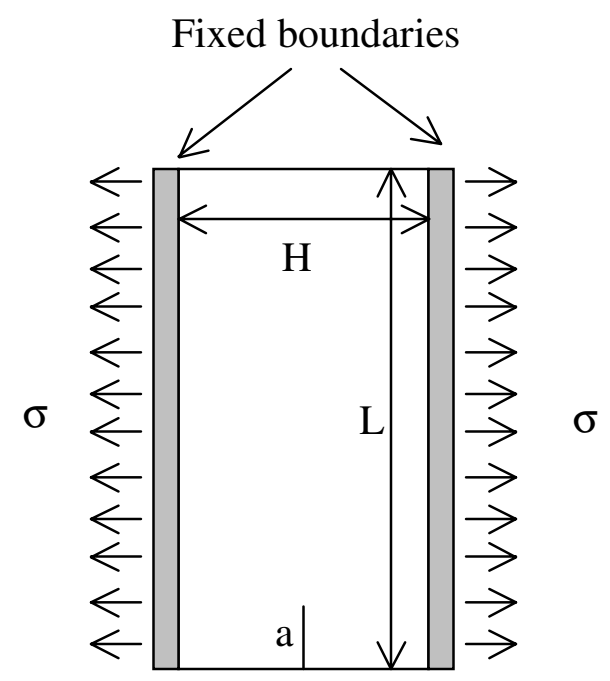

Fig. 3 Sketch of the simulated specimen. 


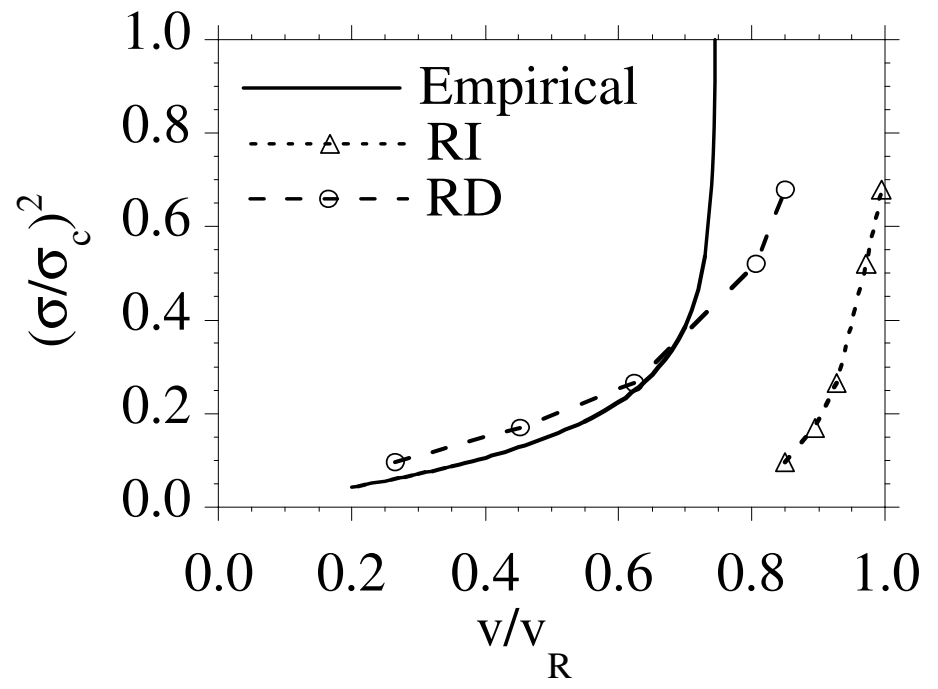

Fig. 4 Empirical fit to experimental data for PMMA together with data from Zhou et al. (2005) for rate-independent (RI) and rate-dependent (RD) models. 


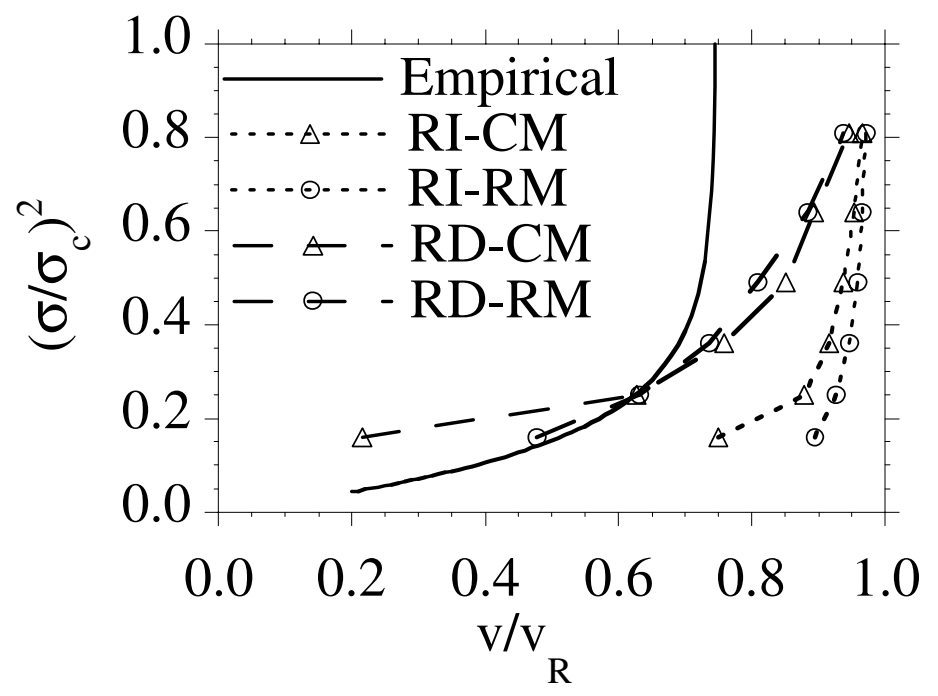

Fig. 5 Empirical fit to experimental data for PMMA together with simulations using rateindependent $(\mathrm{RI})$ and rate-dependent $(\mathrm{RD})$ degradation of the nodal cohesive force with no continuum damage evolution. Also shown are the results for a coarse mesh (CM) and a more refined mesh (RM). 

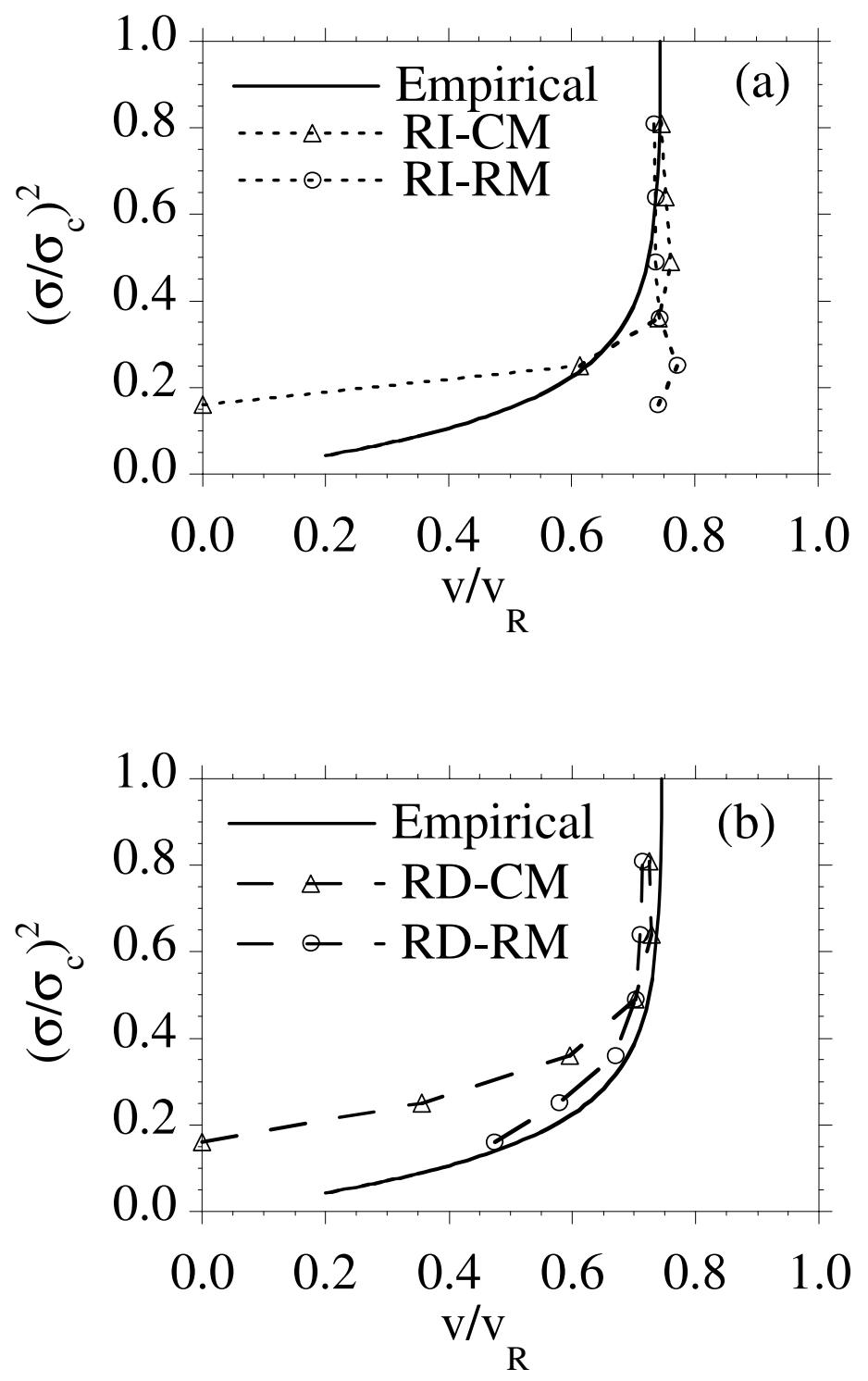

Fig. 6 Empirical fit to experimental data for PMMA together with simulations using rateindependent (RI) and rate-dependent (RD) degradation of the nodal cohesive force with continuum damage evolution. Also shown are the results for a coarse mesh $(\mathrm{CM})$ and a more refined mesh (RM). 


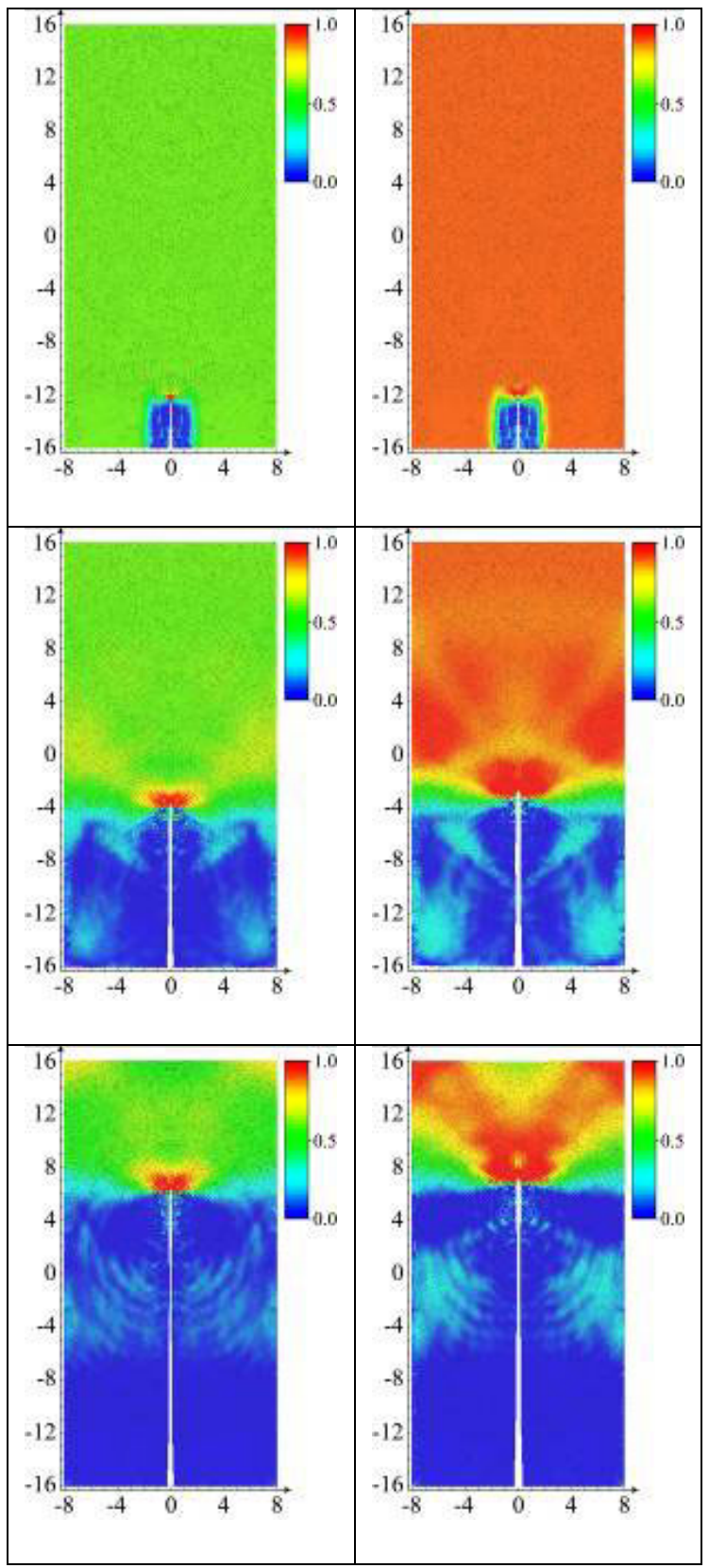

Fig. 7 Maximum principal stress $\sigma_{1} / \sigma_{\mathrm{c}}$ for a single fracture using the refined mesh (RM):

Snapshots at 1, 15, and 30 microseconds after initial loading to $60 \%$ and $90 \%$ of tensile strength (left and right panels, respectively). 


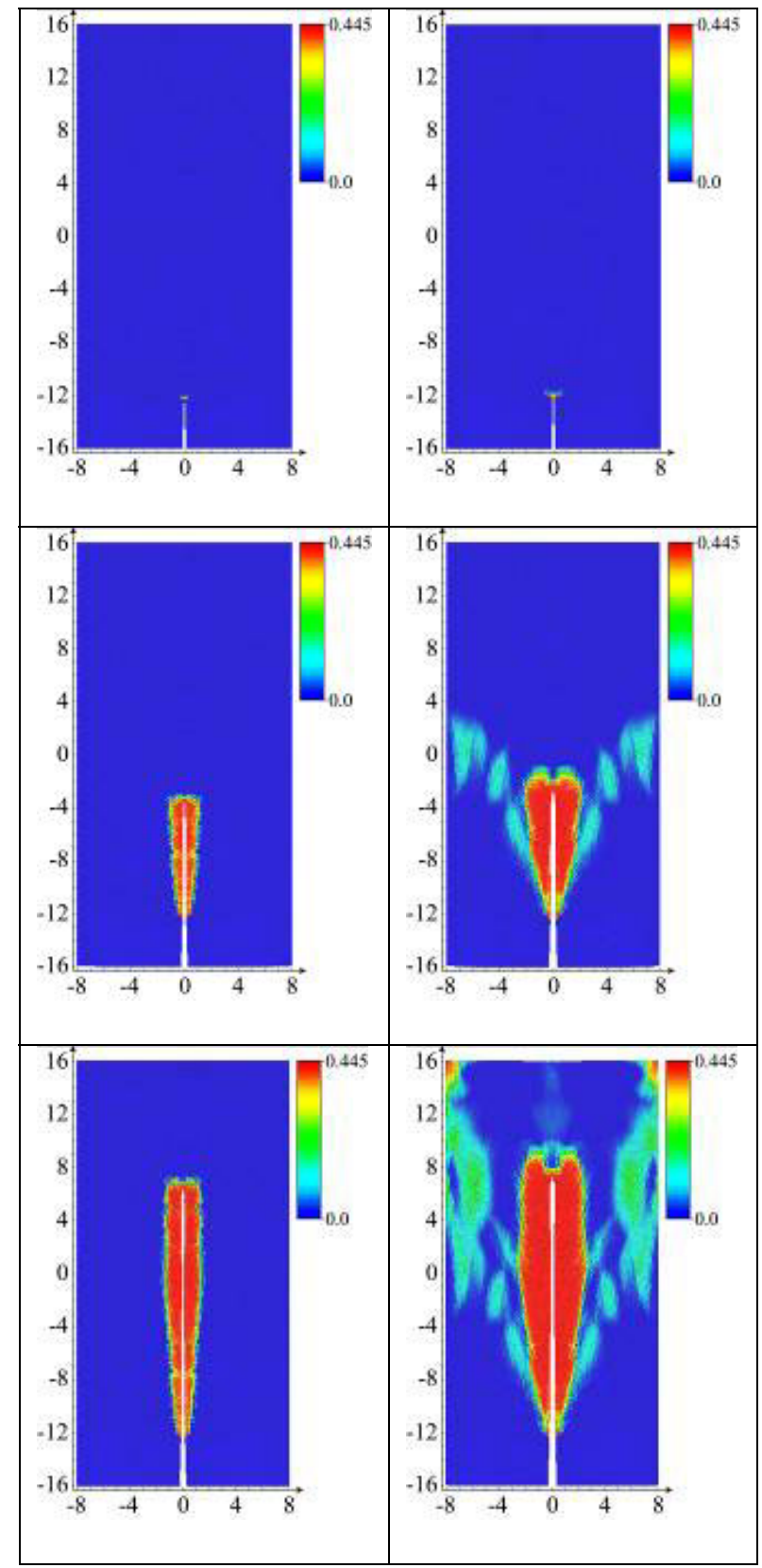

Fig. 8 Damage $\Omega$ for a single fracture using the refined mesh (RM): Snapshots at 1,15 , and 30 microseconds after initial loading to $60 \%$ and $90 \%$ of tensile strength (left and right panels, respectively). 


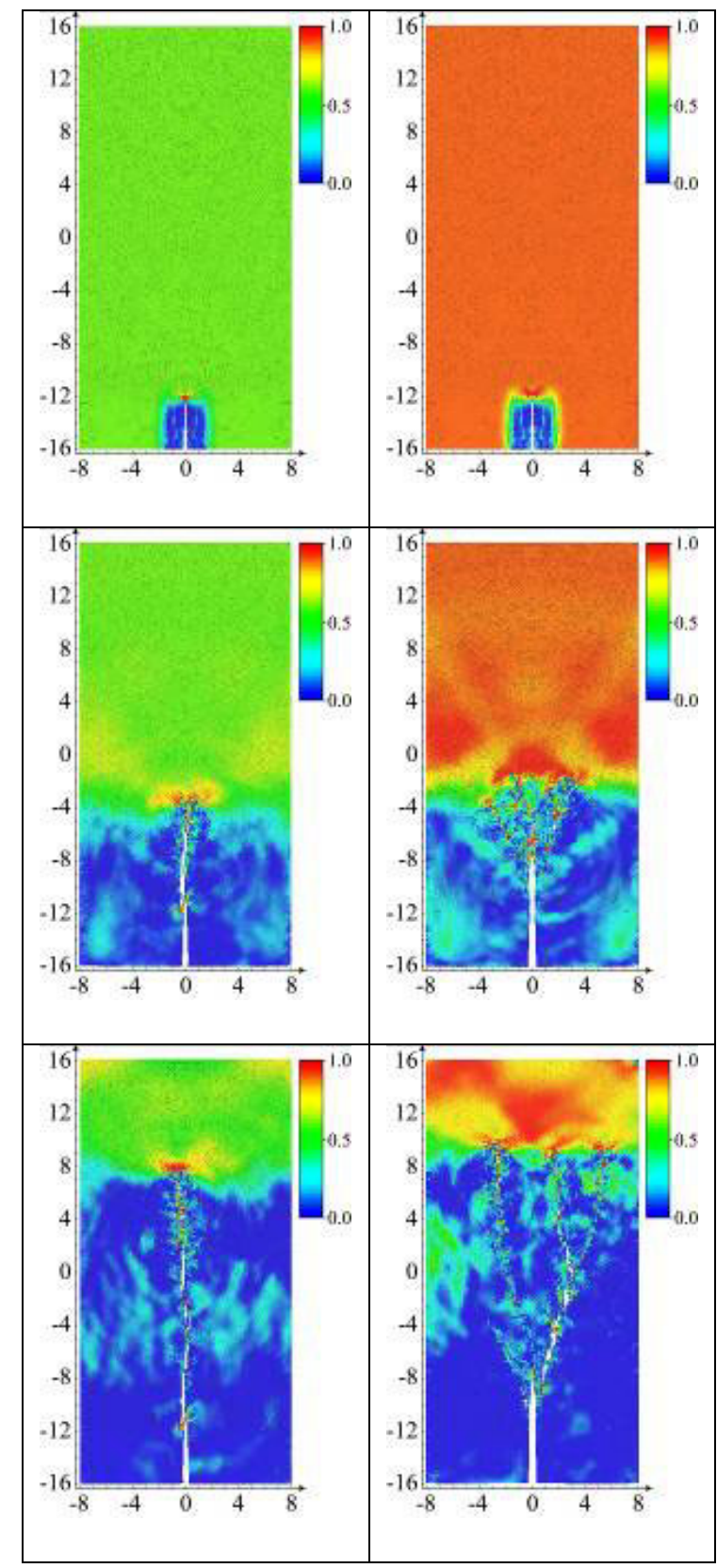

Fig. 9 Maximum principal stress $\sigma_{1} / \sigma_{\mathrm{c}}$ for branching using the refined mesh (RM):

Snapshots at 1, 15, and 30 microseconds after initial loading to $60 \%$ and $90 \%$ of tensile strength (left and right panels, respectively). 


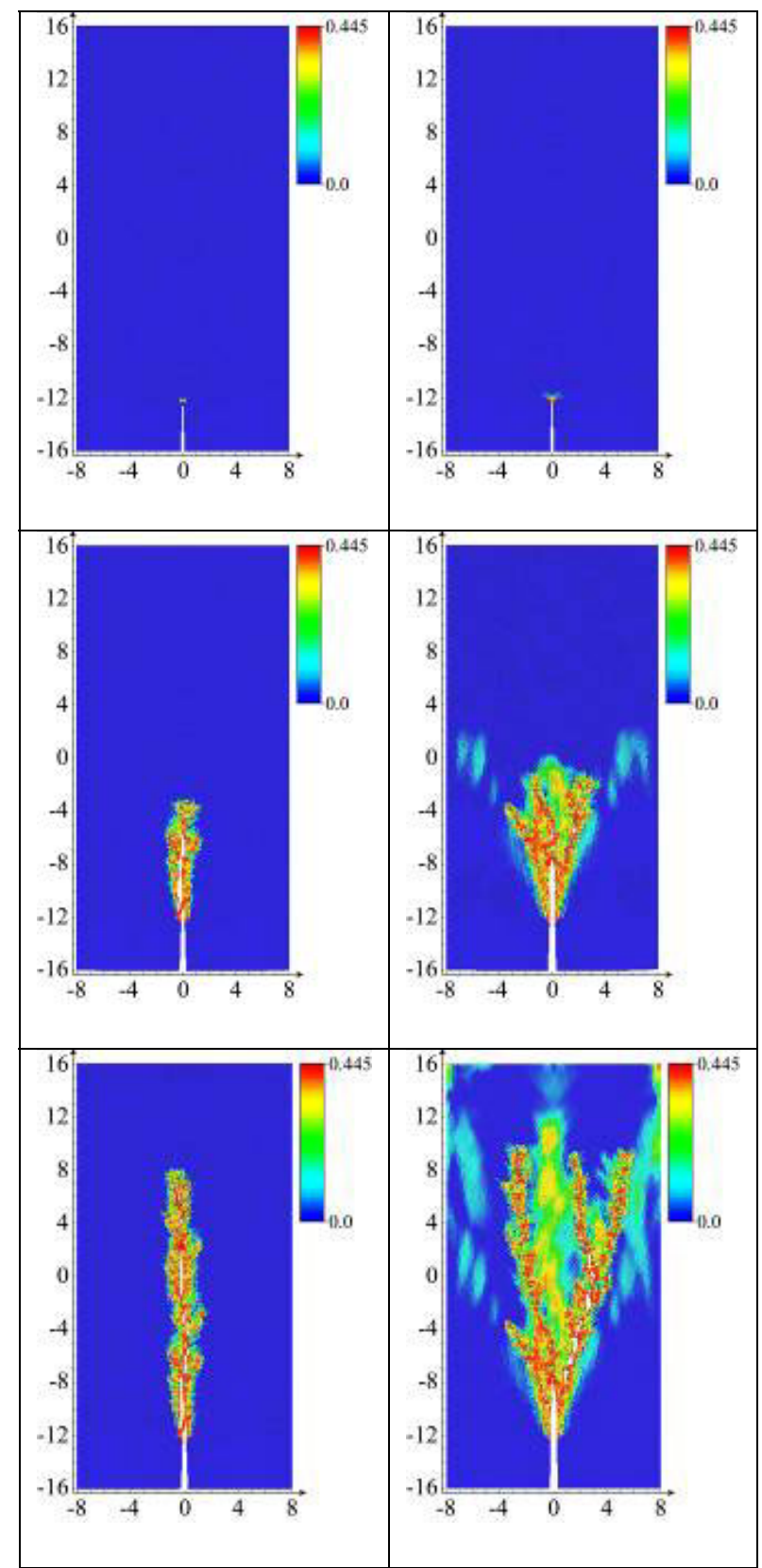

Fig. 10 Damage $\Omega$ for branching using the refined mesh (RM): Snapshots at 1, 15, and 30 microseconds after initial loading to $60 \%$ and $90 \%$ of tensile strength (left and right panels, respectively). 